\title{
Effect of Lifestyle Modification Intervention Program Among Adults Suffering from Osteoarthritis knee.
}

\author{
Eman Shokry Abd Allah ${ }^{1}$, Sabah Abdou Aly Hagrass. ${ }^{2}$, \\ Omaima Ahmed Hassanin, ${ }^{3}$ \\ ${ }^{I}$ (Professor of community health nursing and Head of Gerontological Nursing Department, Faculty of Nursing, \\ Zagazig University, Egypt) \\ ${ }_{2}^{2}$ (lecturer- of community Health Nursing Department- Faculty of Nursing, Egypt) \\ ${ }^{3}$ (B.Sc.N. Zagazig University, Egypt)
}

\begin{abstract}
Osteoarthritis is a common chronic disease affect female more than male, and lead to pain, functional disability, and lower quality of life.

The study aim: to evaluate the effect of lifestyle modification intervention program among adults suffering from osteoarthritis knee.

Design: Quasi-experimental was used.

Setting: the study conducted in out patient orthopedic and rheumatology clinics at Zagazig University. A purposive sample 80 adults females suffered from osteoarthritis.

Tools: of data collection were interview questionnaire to collect data about general characteristic of the study sample, medical history, knee injury and OA scale, lifestyle modification sheet and observation checklist.

Results: revealed $41.3 \%$ of study was obese, and most of them suffered from pain and difficulties in daily activities.

Conclusion: lifestyle modification program improve their pain and stiffness and physical activities. Recommendation: health education about lifestyle modification to all females suffered from $O A$ attended to orthopedic clinics. Further study in other place for early detection and prevention of OA among adults females.

Keywards: Effect, lifestyle modification intervention program, adults, osteoarthritis knee.
\end{abstract}

\section{Introduction}

Osteoarthritis (OA) known as degenerative arthritis or degenerative joint disease or osteoarthrosis, is a group of mechanical abnormalities involving degradation of joints, including articular cartilage and subchondral bone ( Lozada, 2009 ) . The knee is one of the most commonly affected joints. OA is usually a slowly progressive degenerative disease in which the joint cartilage gradually wears away. It most often affects middleaged and older people over 50 years of age, particular in women (Hagglund, et al., 2007 ). Osteoarthritis is the most prevalent form of arthritis in the United States, affecting more than $70 \%$ of adults . Women are affected more than men. It is estimated that $80 \%$ of the population have radiographic evidence of OA, although only $60 \%$ of those will have symptoms ( Lawrence, et al., 2008) .

Osteoarthritis is one of the most frequent causes of physical disability among adults. Stiffness, joint pain, and swelling are the earliest symptoms of osteoarthritis. (Laufer, et al., 2005) .Physical findings include painful limitation of movement, bony crepitus, and, occasionally, joint effusions and joint line or bone tenderness. As the disease progresses, more permanent joint deformities can occur in the forms of contractures, osteophytes, and loss of joint function. Symptoms may include joint pain, tenderness, stiffness, locking, and sometimes an effusion (Silva, et al., 2007). Treatment generally involves a combination of exercise, lifestyle modification, and analgesics. If pain becomes debilitating, joint replacement surgery may be used to improve the quality of life. OA is the most common form of arthritis, and the leading cause of chronic disability (Fary, et al., 2008 ) . Lifestyle modification such as weight loss and exercise for most people with OA, should be the mainstay of their self-management. Moderate exercise leads to improved functioning and decreased pain in people with osteoarthritis of the knee, and weight loss may be an important factor also. Patient education has been shown to be helpful in the self-management of arthritis. It decreases pain, improving function, reducing stiffness and fatigue, and reducing medical usage(Mayo clinic staff, 2006 ).

The community health nurse play an important role to empower the patient and family with teaching knowledge and skills necessary to reach optimal safe performance to manage pain and disability of osteoarthritis ( Bashaw \&Teingstad, 2005 ).

\section{Significance of the Study:}


Osteoarthritis is the most common joint disease in the worldwide. Osteoarthritis is also among the most common causes of pain and disability in older people, and also people younger than age 45. Excess weight or obesity - Obese women are four to five times more likely to have osteoarthritis knee than people of normal weight. Osteoarthritis (OA) is one of the oldest and most common forms of arthritis amongadults females. Known as the "wear-and-tear" kind of arthritis, OA is a chronic condition characterized by the breakdown of the joint's cartilage. The breakdown of cartilage causes the knee bones to rub against each other, causing stiffness, pain and loss of movement and lead to physical function is limited. The treatment depends on the joint but often includes medication, therapeutic exercise, weight reduction and in addition to lifestyle modification is necessary to improve symptoms of osteoarthritis and also lead to improve in general health status, and improve quality of life for these group of patients.

\section{The Aim of the Study:}

The aim of this study is to evaluate the effect of lifestyle modification intervention program among adults suffering from osteoarthritis knee.

\section{Research Hypothesis:}

Using of therapeutic exercises with lifestyle modification intervention have a great effect on relieve pain, stiffness and improving of physical function in patients with osteoarthritis knee after implementing of the lifestyle modification educational program.

\section{Subjects and Methods:}

Research Design:

A quasi experimental study used in the study.

Setting:

The study was conducted at out patient clinics of orthopedic and rheumatology clinics at Zagazig University Hospitals.

Sample:

A purposive sample of 80 adults aged 30 to 50 years females diagnosed with osteoarthritis knee, and all receive pharmacological treatment, and accept to participation in health lifestyle modification.

Tools:

Four tools were used name questionnaire to collect data about general characteristics of the study sample such as age, educational level, and occupation ...etc. Medical history (duration of disease, treatment taken for managing, in addition to calculation of BMI to indicate degree of obesity using the following equations according to ( Castillo- Martinez, et al., 2012) .

\section{BMI = ( weight in kilograms $) /($ height in meter $) 2$}

- Under weight $<18$

- Normal BMI $\geq 18-24.9$

- Over weight $\geq 25-29.9$

- Obese $\geq 30-40$

- Morbid obese > 40

- Knee Injury and Osteoarthritis Outcome Score (KOOS).This tool was developed by Eaa \& Stefan, (2003), consisted of 4 scale to assess the degree of pain, perform of daily activities, patient's feeling.

- Lifestyle knowledge questionnaire, developed by researcher after literature review, to collect data about patient information about pain management, important of body reduction, practice exercises, adherent to treatment.

- Observational sheet to assess participant performance exercise guided by likert scale (2007), and developed by researcher after extensive review.

Methods:

1- Permission to conduct the study was obtained from the administrator of the hospital and head of the department (orthopedic and rheumatology clinic).

2- A purposive sample of 80 adults females suffered from $\mathrm{AO}$ and attended the above mentioned setting were included.

3- Tools of data collection were designed based on recent relevant literature and tested for its content validity and relevance by a Jury consisted of three academic staff in community health nursing from El Zagazig University.

4- The purpose of the study was explained to all participants.

5- Pilot study was conducted on $10 \%$ of the study sample in order to ascertain its clarity and feasibility.

6- Each took $15-25$ minutes to be answered. 
Ethical consideration:

The questionnaire sheet was explained to the participants, verbal consent was obtained from each subject. Privacy was maintained during process of data collection and confidentially.

\section{Statistical Analysis :}

After collection of the data it was revised, coded and fed to statistical software SPSS version 16. The given graphs were constructed using Microsoft excel software. All statistical analysis was done using two tailed tests and alpha error at $\mathrm{P}$ value $<0.05$.

Results :

Table 1: Socio-demographic characteristics of patients in the study sample $(n=80)$

\begin{tabular}{|l|l|l|}
\hline Items & Frequency & Percent \\
\hline Age: & \multicolumn{2}{|l|}{} \\
\hline$<45$ & 48 & 60.0 \\
\hline $45+$ & 32 & 40.0 \\
\hline Range & $30.0-50.0$ \\
\cline { 1 - 2 } Mean \pm SD & $42.2 \pm 5.0$ & \\
\hline Median & 42 & \\
\hline Current marital status: & \multicolumn{2}{|l|}{} \\
\hline Unmarried & 15 & 18.8 \\
\hline Married & 65 & 81.3 \\
\hline Education: & & \\
\hline Illiterate/ Read/write & 16 & 20.0 \\
\hline Basic/secondary & 11 & 13.8 \\
\hline Post/secondary & 35 & 43.8 \\
\hline University & 18 & 22.5 \\
\hline Job: & & \\
\hline Housewife & 33 & 41.3 \\
\hline Working & 47 & 58.8 \\
\hline Crowding index: & & \\
\hline$<2$ & 41 & 51.3 \\
\hline $2+$ & 39 & 48.8 \\
\hline
\end{tabular}

Table 1 revealed the mean age of the study female was $42.2 \pm 5.0$ years and $43.8 \%$ of their had postsecondary education, while $22.5 \%$ port university degree.

Table 2: Patients' body mass index (BMI) before and after the intervention

\begin{tabular}{|c|c|c|c|c|c|c|}
\hline \multirow[t]{3}{*}{ Items } & & & & Time & \multirow[t]{3}{*}{$\mathrm{X}^{2}$ test } & \multirow[t]{3}{*}{ p-value } \\
\hline & \multicolumn{2}{|c|}{$\operatorname{Pre}(\mathrm{n}=80)$} & \multicolumn{2}{|c|}{ Post $(\mathrm{n}=80)$} & & \\
\hline & No. & $\%$ & No. & $\%$ & & \\
\hline \multicolumn{7}{|l|}{ BMI: } \\
\hline Overweight $(<30)$ & 8 & 10.0 & 10 & 12.5 & & \\
\hline Obese $(30-<35)$ & 33 & 41.3 & 34 & 42.5 & 0.36 & 0.84 \\
\hline Morbid obese $(35+)$ & 39 & 48.8 & 36 & 45.0 & & \\
\hline Range & \multicolumn{2}{|c|}{$27.7-44.1$} & \multicolumn{2}{|c|}{$27.7-44.1$} & & \\
\hline Mean \pm SD & \multicolumn{2}{|c|}{$35.0 \pm 3.6$} & \multicolumn{2}{|c|}{$34.6 \pm 3.6$} & $\mathrm{U}=0.56$ & 0.46 \\
\hline Median & \multicolumn{2}{|r|}{34.8} & \multicolumn{2}{|r|}{34.6} & & \\
\hline
\end{tabular}

(*) Statistically significant at $p<0.05$ (U) Mann-Whitney test

Table 2 demonstrates no statistically significant differences between pre and post-intervention values. 


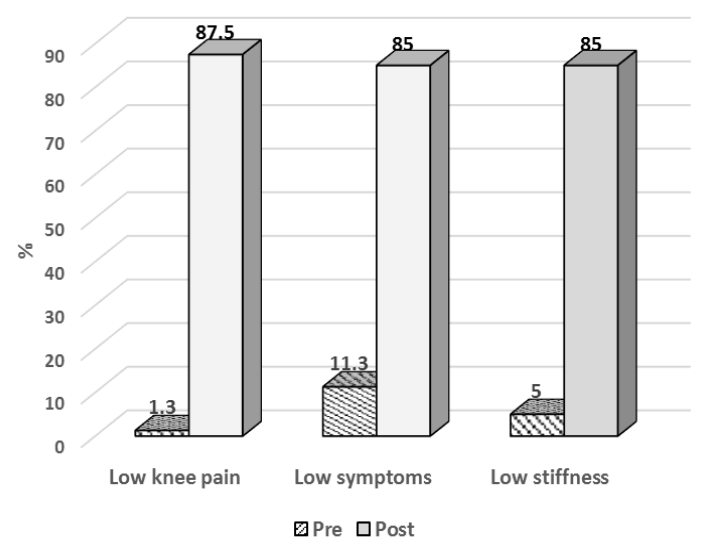

Figure 1: Total knee pain, symptoms, and stiffness as reported by patients before and after the intervention

Figure 1 illustrates statistically significant improvements in patients' total knee pain, symptoms/signs, and stiffness after the intervention $(\mathrm{p}<0.001)$.

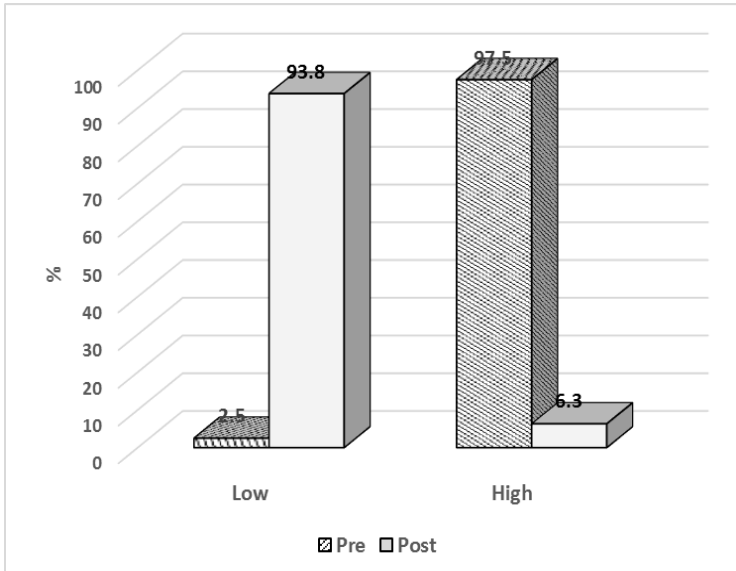

Figure 2: Patients' total reported problems with performance of daily life activities (DLA) before and after the intervention

Figure 2 portary improvement in total patient performance of their daily activities post intervention program ( $\mathrm{P}$ $=<0.001)$.

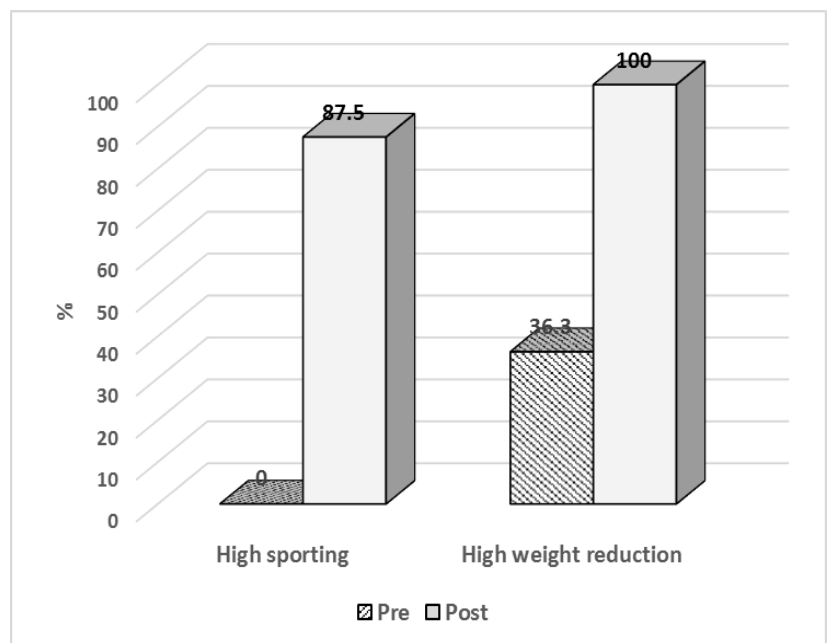

Figure 3: Total patients' behaviors related to sporting and weight reduction before and after the intervention Figure 3 illustrates statistically significant improvements in patients' total sporting and weight reduction behaviors after the intervention $(\mathrm{p}<0.001)$. 


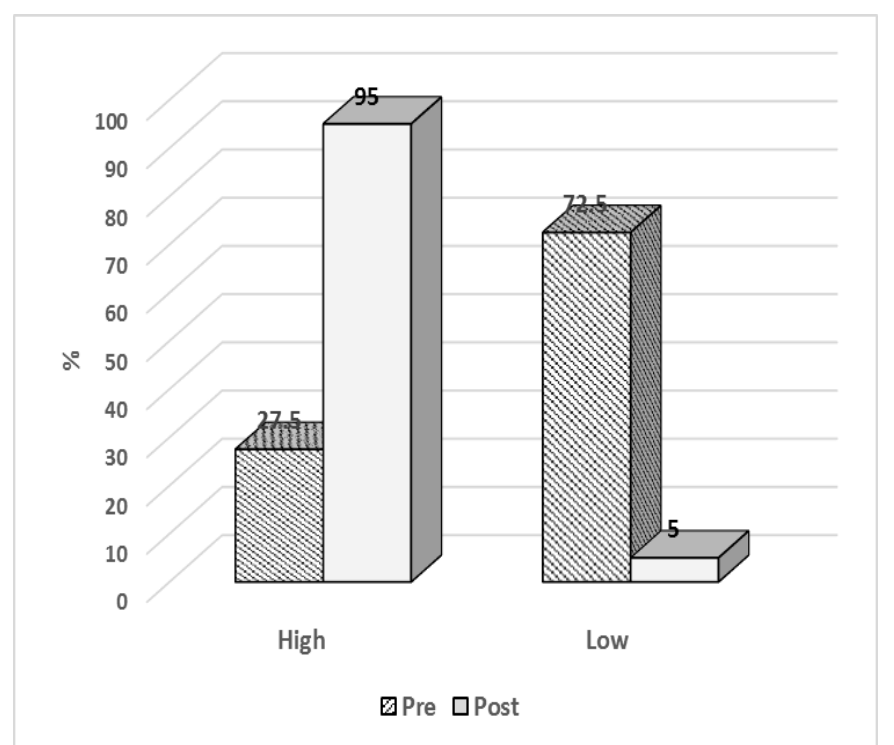

Figure 4: Patients' total behavior before and after the intervention In sum,

Figure 4 demonstrates statistically significant improvement in patients' total behavior after the intervention ( $\mathrm{p}$ $<0.001)$.

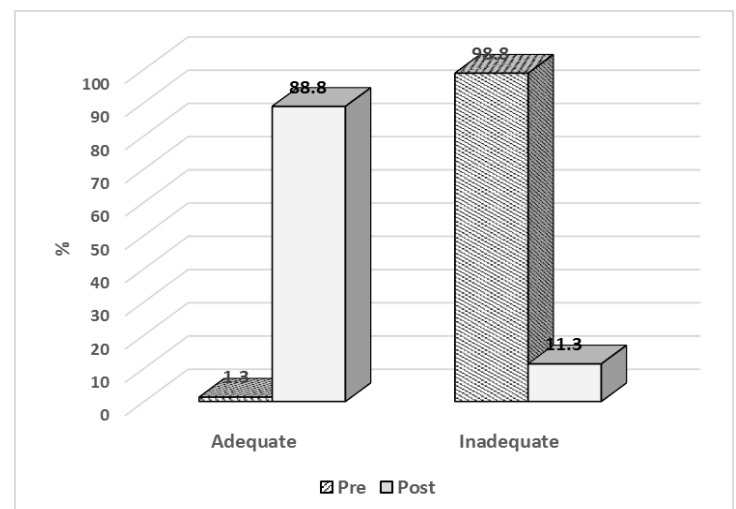

Figure 5: Patients' total practice of exercises before and after the intervention

Figure 5 illustrates the presence of statistically significant improvement in patients' total practice of exercises after the intervention $(\mathrm{p}<0.001)$.

Table 3: Best fitting multiple linear regression model for the behavior score

\begin{tabular}{|l|l|l|l|l|l|l|l|}
\hline & \multicolumn{2}{|l|}{$\begin{array}{l}\text { Un-standardized } \\
\text { Coefficients }\end{array}$} & \multirow{2}{*}{$\begin{array}{l}\text { Standardized } \\
\text { Coefficients }\end{array}$} & \multirow{2}{*}{ t-test } & p-value & \multicolumn{2}{l|}{$\begin{array}{l}\text { 95\% Confidence } \\
\text { Interval for B }\end{array}$} \\
\cline { 2 - 6 } & $\mathrm{B}$ & Std. Error & & & & Lower & Upper \\
\hline Constant & 0.78 & 0.20 & & 3.87 & $<0.001$ & 0.38 & 1.18 \\
\hline Education & 0.07 & 0.02 & 0.15 & 3.59 & $<0.001$ & 0.03 & 0.10 \\
\hline Crowding index & -0.51 & 0.13 & -0.16 & 3.84 & $<0.001$ & -0.77 & -0.25 \\
\hline Intervention & 1.37 & 0.06 & 0.84 & 21.93 & $<0.001$ & 1.25 & 1.50 \\
\hline
\end{tabular}

$\mathrm{r}$-square $=0.77$

Model ANOVA: $\mathrm{F}=175.93, \mathrm{p}<0.001$

Variables entered and excluded: age, job, marital status, residence, BMI, duration of illness

It shows that the intervention was a statistically significant positive predictor of this score, in addition to the level of education. On the contrary, a higher crowding index was a negative predictor.

Table 4: Best fitting multiple linear regression model for the practice score

\begin{tabular}{|r|r|r|r|r|r|}
\hline & Un-standardized & Standardized & t-test & p-value & $95 \%$ Confidence \\
\hline
\end{tabular}


Effect of Lifestyle Modification Intervention Program Among Adults Suffering from

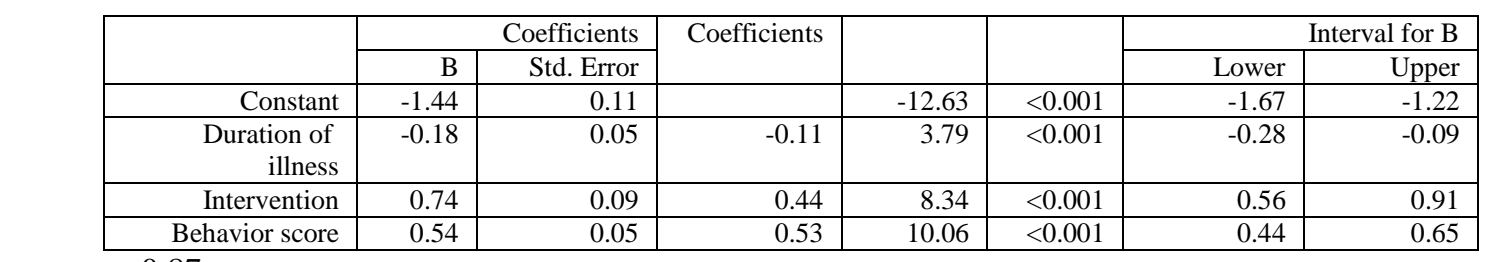

r-square $=0.87$

Model ANOVA: $\mathrm{F}=357.68, \mathrm{p}<0.001$

Variables entered and excluded: age, education, job, marital status, residence, crowding index, BMI

Table 4 demonstrates that the intervention was a statistically significant positive predictor of this score, in addition to the behavior score.

\section{Discussion}

Osteoarthritis (OA) is a common type of arthritis in which there is a gradual loss of cartilage from the joints. Common osteoarthritis symptoms include pain, stiffness, some loss of joint motion, and changes in the shape of affected joints Sridhar, et al.,(2012).The treatment depends on the joint but often includes medication and exercise. There is no special osteoarthritis diet, but lifestyle modification and weight loss may improve symptoms of osteoarthritis Ayres, et al., (2012) .The aims of the present study were to evaluate the effect of lifestyle modification intervention program among adults suffering from osteoarthritis knee.

The current study reveled that, slightly less than two-third of the patients aged less than 45 years, and more than one third of the adults female their age was more than 45 years old, with mean $42.2+5.0$ years, this finding was goes in the same line with an Egyptian study conducted by Life Science Journal, (2011), which supported this study by found that, osteoarthritis usually occurs in any age and age exemplifies one of the most important risk factors for osteoarthritis. Evidence of joint changes occurs at approximately age 40 and changes continue to occur to the extent that the majority of elderly persons reveal joint changes indicative of osteoarthritis and occurs more often in women than in men, (although more men are affected when osteoarthritis occurs in people younger than age 45).

The present results revealed that Females were more affected with knee(OA), because female's hormones lead to the development of OA. In addition women are more susceptible to obesity than men which are another important risk factor for OA. This is consistent with previous reports of Sudo, et al., (2008) and Sanjeev, \& Michael, (2012) who revealed that knee OA was more common among females than males.Concerning the level of education, the present study revealed that post/secondary was prevailing among less than half of the patients, this finding in the same line with Kim et al., (2010) who stated that knee OA is significantly greater with a good level of education. Also this is in agreement with The Center for Disease Control and Prevention (CDC, 2010) which reported that the incidence of knee OA in people with high education more than in people of illiteracy.

As regard body mass index the study finding revealed one ten of study women over weight, while less than half obese or morbid obese. It considered an important variable related to OA is the body mass index. Increase adult weight due to bad nutritional habits, and lack of activities in addition to hormonal change associated with menopause all this lead to decrease bone density. This finding was in same line with study done in Egypt by $\boldsymbol{A} \boldsymbol{b d}-\boldsymbol{A l} \boldsymbol{A t t y}$, (2011) found that bone mineral density had statistically significant positive correlation with body and body mass index, on the same line with Wasan and Abdulameer, (2014) who study done in United Arab Emirate found that body mass density was influenced significantly by all independent variables (age, gander and BMI ) together in the osteoarthritis, so all variables together are considered as risk factors of OA . A similar finding was reported in another Iranian study Salamat, et al., (2013) who reported that female who over weight and obese were more likely to have OA. Although denied with study done in London by Reid, (2013) found that there is no clear explanation for the mechanism or BMI on bone structure. However, it has been clarified that when weight is moderately high, consequently there is more weight - burden on joints and knee joints . The results of the study revealed statistically improvement in patients total knee pain, symptoms, and stiffness after the intervention. These findings come in consistent with The Centers for Disease Control and Prevention, (CDC, 2010) finding the onset of osteoarthritis symptoms develop slowly and may be stable for many years. Osteoarthritis symptoms may occur in any joint; however, the joints most often affected are the knees, hips, hands, and spine. And joints need motion to stay healthy. Long periods of inactivity cause the arthritic joint to stiffen and the adjoining tissue to atrophy (waste away). A moderate exercise program that includes low-impact aerobics, power and strength training has benefits for patients with osteoarthritis. And when combination between physical therapy and weight reduction they can relieve osteoarthritis symptom for long period.The finding of the present study indicates all of the study females almost all of them were having such problem associated with daily activities, 
but post intervention significant reduction in all participants daily activities. This is accordance with these findings Mauck and Clarke, (2006) who study in USA demonstrated that effective health education program enhance quality of life through educate people about important of bone health, by promoting bone healthy nutrition, promoting regular physical activities. In addition to public sport programs, periodical visit to physician all can promote daily activity.

Also the finding of the present study demonstrates that almost all of the patients were having problems with performance of sporting and recreation activities before intervention this problem decreased post intervention. This results is expected since the lack of physical activities is known as risk factors for OA and practicing exercise lead to improve this condition. This result stand on line with Etemadifar, et al., (2013) in Iran emphasized that exercise has an important on bone health. Immobilization is an important cause of bone loss and should where ever possible be avoided. The amount of exercise that is optimal for skeletal health in patient with OA is not known, but regular bearing exercise forms on integral component of management and should be tailored according to the needs and fitness of the women. Similar finding were reported by Ragucci, and Shrader, (2011) who study in Cambridge mentioned that immobilization lead to rapid loss of bone, patient confined to bed may loss up to $1 \%$ of trabecular bone per week, cortical bone is lost at some what slower rate, and weight- bearing bone being the most affected, resumption of normal weight bearing activity which gradually restores bone health. The implementation of the present study program of modification of lifestyle by practice exercise, reduction of body weight lead improvement in practice of physical exercise among adults female which improve and reduction knee pain and problem. In agreement with the finding a study done in Brazil Burke, et al., ( 2012 ) found that adults women who practice exercise regularly for at least 30 minutes of moderate exercise or physical activity at least 5 time per week improved knee pain .

Similarly, Orr, et al., (2008) who conducted study in Australia found adults multi component exercise. Programs incorporating a combination of weight- bearing impact exercise ( jogging, stair - climbing, walking, jumping activities) and progressive resistance training were most effective for maintaining bone health . Moreover physical exercise is a lifestyle factor that has been associated with numerous health benefits and is likely to contribute to the prevention of OA. Rizzoli, et al., (2012) further more, exercise program have a key role in management of OA among adults and can results in positive benefits. It can provide overall increase in strength, flexibility and balance and improve quality of life particularly in the domain of physical function such balance and pain relief Papaioannou, et al., (2010).The current study finding revealed that the lifestyle modification program was statistically significant positive predictor in change of the participant behavior, and practice score, in addition to level of education. This results indicates the most influential factor on the behavior and practice score change was the lifestyle modification program. This finding proved the hypothesis of the study. This mean treatment generally involves a combination of exercise, lifestyle modification and analgesics (Fary, et al., 2008) change patient's behavior and practice.

\section{Conclusion}

The lifestyle modification intervention program provided to females with OA improved their total daily activities and reduce pain.

\section{Recommendation}

1-Booklet contain all information about osteoarthritis knee, causes, prevention and management should be available in each health care setting provided care for OA .

2-2- Instruct females about important of control their body weight and practice exercises.

\section{References}

[1]. Abd- Al Atty, M.F., (2011) : Regional fat, weight and osteoarthritis in adults women in Egypt. Eastern Mediterranan Health Journal; 17 (11): 850 - 854 .Ayres, E., Warmington M, and Reid M.C.( 2012) : Managing chronic pain in young adults: 6 steps to overcoming medication barriers ; S16-21.

[2]. Bashaw, R.T.,\&Teingstad, E.M. (2005): Rehabilitation of the osteoarthritic patient: focus on the knee. Clinical Sports Medicine; 24 : 101-131.

[3]. Burke, T.N., Franca, F.G., Meneses and Marques, A.P., (2012): Postural control in adult woman with osteoarthritis comparison of balance, strengthening and stretching exercises. A randomized controlled trial. Clinical Rehabilitation 210 (11) : $1021-1031$.

[4]. Eaa, M. Roos, and Stefan, Lohmander. (2003): The Knee injury and Osteoarthritis Outcome Score (KOOS): from joint injury health equal life outcome journal. Volume. 1 page 64.

[5]. Etemadifar, M.R., Nourian, S.M., Fereidan-Esfahani, M., Shemshak, H., and Zareadh, M., (2013) : Relationship of knowledge about osteoarthritis with educational level, and life habits world J orthopedic; 4 (3) : $139-143$.

[6]. Fary, R.E., Carroll, G.J., Briffa, T.G., Gupta, R., and Briffa, N.K. (2008): The effectiveness of pulsed electrical stimulation in the management of osteoarthritis of the knee: a protocol for a randomised controlled trial. BMC Musculoskeletal Disorders; 9:18.

[7]. Hagglund, K.J., Clark, M.J., Hilton, S.A., and Hewett, J.E. (2007): Access to healthcare services among persons with osteoarthritis and rheumatoid arthritis. Am J Phys Med Rehabil; 84(9): 702-711.

[8]. Kim, I., Kim, H., and Seo, Y. (2010) : The Prevalence of Knee osteoarthritis in Elderly Community Residents in Korea. Jornal of Korean Medicine Science; 25: 293 - 8. Available at http://www.ncbi.nlm.nih.gov/pubmed/ 20119586. 
[9]. Laufer, Y., Zilberman, R., Porat, R., and Nahir, A.M. (2005): Effect of pulsed short-wave diathermy on pain and function of subjects with osteoarthritis of the knee: a placebo-controlled double-blind clinical trial. Clinical Rehabilitation; 19: $255-263$.

[10]. Lawrence, R.C., Felson, D.T., and Helmick, C.G. (2008): Estimates of the prevalence of arthritis and other rheumatic conditions in the United States. Part II. Arthritis Rheum; 58 (1) : $26-35$.

[11]. Life Science Journal, (2011): Comparison between uses of therapeutic exercise and heat application on relieve pain, stiffness and improvement of physical function for patient with knee osteoarthritis Available at Website: http: // www. lifesciencesite.com.

[12]. Likert Scale. (2007): Available at Website:

[13]. http: // www.hkadesigns.co.uk/websites / msc / reme / likert.htm> Accessed on, [April 20, 2012]

[14]. Lozada, C.J. (2009): Management of osteoarthritis. In GS Firestein., Kelley's Textbook of Rheumatology. Philadelphia: Saunders Elsevier 8th ed. Vol. 2, pp. $1563-1577$.

[15]. Mauck, K.F., and Clarke B.L., (2006) : Diagnosis, screening, prevention and treatment of osteoarthritis. Mayo Clinic proc ; 81(5) : $662-672$.

[16]. Mayo clinic staff. (2006): osteoarthritis, Available at Website: http://www.mayo clinic. Com / health / OA / D500019 / Accessed on, [February 14, 2012].

[17]. Orr, R., Raymond, J., and Fiatarone Singh, M., (2008) : Efficacy of progress resistance training on balance performance in adults; A systematic review of randomized controlled trails. Sports Med. 38 (1): $317-343$.

[18]. Papaioannou, A., Morin, S., Cheung, A.M., Brown, J.P., and Feldman, S., (2010) : Clinical practice guidelines for the diagnosis and management of osteoarthritis in Canada Summary. CMAJ; $23: 182$ (17) : $1864-73$.

[19]. Ragucci, K.R., and Shrader, S.P., (2011) : Osteoarthritis treatment; An evidence - based approach. Journal of Gerontological Nursing 37(7): $17-22$.

[20]. Reid, I., (2013) : Relationships between Body fat and Bone Mass in : P. Burckardt B., Bawson - Hughes C.M., WEAVER, eds, Springer London, PP $83-92$.

[21]. Rizzoli, R., Bianchi, M.L., Gavabedian, M., Morenol, A., (2012) : Maximizing bone mineral mass gain during growth for the prevention of fracture in the adolescents and elderly Bone : $46(2): 294-302$.

[22]. Salamat, M.R., Salamat A.H., Abedi I., and Janghorbaui M., (2013) : Relation between weight, body mass index and Bone Mineral Density in men referred for Dual- Energy X- ray Absorptiometry scan in Isfahan, Iran Journal osteoporosis; 20(5) : 9 - 63

[23]. Sanjeev Sabharwarl, MD., Michael, Z., Impact of Obesity on Orthopaedics (2012) : The Journal Of Bone And Joint Surgery, 94, 1045-1052 ( A1).

[24]. Silva, A.L.P., Imoto, D.M., and Croci, A.T. (2007): Comparison of cryo therapy, exercise and short waves in knee osteoarthritis treatment. Acta Ortopedica Brasileria; 15: $204-209$.

[25]. Sridhar MS, Jarrett CD, Xerogeanes JW, Labib SA. ( 2012) : Obesity and

[26]. symptomatic osteoarthritis of the knee, 94(4): 433 - 440 Sudo A, Miyamoto N, Horikawa K, et al (2008): Prevalence and risk factors for knee osteoarthritis in elderly Japanese men and women Journal of Orthopedic Science; 13(5) 413-18.

[27]. The Centers for Disease Control and Prevention, (2010): "Osteoarthritis and you" Available at: http:// www. c d c. gov/ Features/ Osteoarthritis Plan/ Accessed on, [February 20, 2012].

[28]. Wasan, A.M., and Abdulameer, (2014): The correlation of body mass index, age, gender with Bone Mineral Density in osteoarthritis, osteoporosis, A study in the United Arab Emirates, clinical Medicine and diagnosis, 4(3): 42 - 54 . 